\title{
Enseñanza, desigualdades y reconfiguración del trabajo docente en escuelas secundarias durante la pandemia
}

\section{Teaching, inequalities and reconfiguration of teaching work in secondary schools during the pandemic}

\author{
Arroyo, Mariela; Corvalán, Tatiana; Felicioni, Silvina Leonor; \\ Merodo, Alicia
}

Mariela Arroyo Sobre la autora marroyo@campus.ungs.edu.ar Universidad Nacional de General Sarmiento (UNGS), Argentina

Tatiana Corvalán Sobre la autora tcorvala@campus.ungs.edu.ar Universidad Nacional de General Sarmiento (UNGS), Argentina

Silvina Leonor Felicioni Sobre la autora sfelicio@campus.ungs.edu.ar Universidad Nacional de General Sarmiento (UNGS), Argentina

Alicia Merodo Sobre la autora alimerodo@gmail.com Universidad Nacional de General Sarmiento (UNGS), Argentina

\section{Itinerarios educativos \\ Universidad Nacional del Litoral, Argentina ISSN: $1850-3853$ \\ ISSN-e: 2362-5554 \\ Periodicidad: Anual \\ vol. 1, núm. 14, 2021 \\ revistadelindi@fhuc.unl.edu.ar}

Recepción: 15 Noviembre 2020

Aprobación: 26 Julio 2021

URL: https://doi.org/10.14409/ie.2021.14.e0003

Esta obra está bajo una Licencia Creative Commons AtribuciónNoComercial-CompartirIgual 4.0 Internacional.
Resumen: Este artículo presenta los resultados preliminares de una investigación cualitativa que aborda la reconfiguración de la enseñanza y del trabajo docente en escuelas secundarias de la Región Educativa IX de la provincia de Buenos Aires, en el contexto de interrupción de las clases presenciales en el marco de la pandemia y la consecuente política de continuidad pedagógica. Se analiza cómo la pandemia dejó en evidencia los mecanismos de diferenciación social y sus efectos en la profundización de las desigualdades educativas. A través de entrevistas grupales se relevaron las decisiones que tomaron inspectores, directivos y docentes de escuelas secundarias, para sostener el vínculo pedagógico en un escenario que interrumpe el «hacer escuela en presencia». Estas decisiones dan cuenta del proceso de reapropiación de la política en las escuelas bajo realidades simbólicas y materiales desiguales. Las reapropiaciones están condicionadas por la gramática de la escolaridad, el modelo organizacional y la configuración histórica del trabajo docente en la escuela secundaria (la estructura del puesto de trabajo, los saberes docentes disponibles). El cambio de las condiciones de escolarización, dado el pasaje de la enseñanza presencial a distintas formas de enseñanza no presencial, tensionaron y alteraron disposiciones y saberes poniendo al descubierto nuevas desigualdades

Palabras clave: enseñanza, escuela secundaria, trabajo docente, desigualdad educativa, continuidad pedagógica.

Abstract: This article presents the results of a qualitative research that addresses the reconfiguration of teaching and teaching work at secondary schools in a region of the province of Buenos Aires after the interruption of in-person classes during the pandemic and as a consequence of pedagogical continuity policies. The way in which the pandemic revealed the mechanisms of social differentiation is analyzed considering its effects on the deepening of educational inequalities. By means of group interviews, this research collected the decisions of head teachers and teachers of secondary schools to preserve pedagogical links in a scenario that interrupts 'doing inperson school.' These decisions account for a process of a particular appropriation of policies in each school under unequal symbolic and material realities. Appropriations are conditioned by the grammar of 


\begin{abstract}
schooling, the organizational model, and the historical configuration of teaching work (the structure of the job, the available teaching knowledge) at secondary school. The change in schooling conditions -given the switch from in-person teaching to different forms of nonface-to-face teaching-tensed and altered dispositions and knowledge, thus exposing new inequalities.
\end{abstract}

Keywords: teaching, secondary school, teaching work, educational inequality, pedagogical continuity.

\section{Punto de partida}

El presente artículo propone abordar la reconfiguración de la enseñanza y del trabajo docente en escuelas secundarias de la Región Educativa IX de la provincia de Buenos Aires, ${ }^{1}$ en el contexto de interrupción de las clases presenciales como medida preventiva frente a la pandemia y la consecuente política de continuidad pedagógica. En este marco, se analiza el modo en que la pandemia dejó en evidencia las desigualdades sociales y su relación con la desigualdad educativa. Históricamente el sistema educativo ha participado tanto de la legitimación como de la interrupción de estos procesos de desigualdad (o de la correspondencia entre desigualdad social y educativa). En el actual contexto, diferentes grupos sociales disponen de distintos recursos (materiales y simbólicos) para desplegar estrategias frente a la pandemia y frente a la escolaridad no presencial. Ante esa situación, quedan expuestas con mayor crudeza las diversas posibilidades de cumplimiento del derecho a la educación, haciéndose visible el papel central que tiene la escuela en su formato presencial como espacio de sociabilidad y distribución de conocimiento Por otra parte, es importante señalar que estas diferentes posibilidades y estrategias se expresan también en las condiciones que asume el trabajo de los docentes para seguir llevando a cabo la enseñanza.

Para la reconstrucción de las características del trabajo de enseñar, en este trabajo, consideramos las decisiones que fueron tomando inspectores, directivos y docentes de cuatro escuelas secundarias de la mencionada región educativa, en particular en torno al sostenimiento del lazo pedagógico, en un escenario que interrumpe el «hacer escuela en presencia». Estas decisiones dan cuenta de un proceso de resignificación y apropiación de la política de continuidad pedagógica en las escuelas, bajo realidades simbólicas y materiales particulares y desiguales. Estas apropiaciones están condicionadas por la gramática de la escolaridad, el modelo organizacional y la configuración histórica del trabajo docente (la estructura del puesto de trabajo, los saberes docentes disponibles). El cambio de las condiciones de escolarización tensiona y altera disposiciones y saberes.

A través de la Resolución $N^{\circ}$ 108/20 del Ministro de Educación de la Nación se establece la suspensión de clases presenciales en todos los niveles educativos. ${ }^{6}$ Así, alrededor de 10 millones de estudiantes y casi 900 mil docentes de nivel inicial, primario y secundario se vieron afectados por esta decisión. En función del nuevo escenario, las instancias de gestión del sistema (a nivel nacional y jurisdiccional) elaboraron orientaciones de política educativa. Un primer mojón 
fue el establecimiento de la continuidad pedagógica ${ }^{3}$ y las orientaciones para su «implementación», con prioridad en el cuidado y preservación de los vínculos. ${ }^{4}$ La suspensión de las clases presenciales y el retraimiento del «hacer escuela» al ámbito del hogar, puso en jaque supuestos fundamentales de la gramática escolar y de la organización de la enseñanza en la educación secundaria.

Para cumplir con este objetivo, el Ministerio de Educación de la Nación (Secretaría de Educación), aprobó el Programa Seguimos Educando que desarrolla una línea de producción de material impreso con contenidos y actividades escolares y una agenda analógica que incluye catorce horas de televisión y siete horas de programación radial diarias. A su vez, se ha gestionado la navegación gratuita de la plataforma Seguimos Educando y se distribuyeron 135.000 notebooks y tablets. En la Provincia de Buenos Aires se puso a disposición la plataforma Continuemos estudiando, se elaboraron materiales y lineamientos para el trabajo en las escuelas, con foco en el cuidado del vínculo y las nuevas modalidades de evaluación y seguimiento. ${ }^{5}$

La principal estrategia para la continuidad pedagógica utilizada por las escuelas ha sido el contacto mediado por las nuevas tecnologías (plataformas, correo electrónico, redes sociales, WhatsApp, Facebook), tanto para establecer la comunicación con los estudiantes como para hacer posible la enseñanza. La mediación de la tecnología requiere de dispositivos y de saberes tecno pedagógicos y produce nuevas lógicas en las relaciones sociales y pedagógicas que ponen en evidencia nuevas desigualdades y el recrudecimiento de las preexistentes.

Nos interesa analizar las apropiaciones institucionales de los lineamientos de la política educativa a partir de las visiones y condiciones aludidas por los actores institucionales -inspectores, directivos, profesores-. Abordaremos las características que asume la escolarización desde la interrupción de las clases presenciales, a partir de la «experiencia escolar» que se inaugura desde y en los hogares, teniendo en cuenta cómo se pone de manifiesto la continuidad y discontinuidad de la estructura organizacional de la escuela secundaria en este nuevo escenario a partir de las siguientes dimensiones: 1) reconfiguración de la enseñanza y cambios en las condiciones de trabajo docente; 2) relaciones entre desigualdad educativa y sus efectos en la experiencia escolar.

\section{Algunas notas metodológicas}

Este artículo es producto de un trabajo de investigación exploratorio de corte cualitativo realizado con el propósito de reconstruir el proceso de escolarización en educación secundaria durante la pandemia, puesto en diálogo con la historicidad organizativa, estructural, cultural y laboral del nivel.

El material empírico considerado fue relevado en la primera mitad del año 2020. En esta primera etapa del trabajo de campo, se realizaron entrevistas grupales a dos inspectores, cinco directivos y diez profesores de 4 escuelas de la Región IX de la Provincia de Buenos Aires. La muestra de establecimientos fue intencional $y$, aunque no tiene pretensiones de representatividad, se seleccionaron escuelas con distintas características para dar cuenta de la heterogeneidad de experiencias en el nivel educativo de la región. En este sentido, se seleccionó una escuela secundaria con una larga historia en uno de los distritos 
de la región y con una elevada matrícula (alrededor de 1000 estudiantes), dos escuelas de reciente conformación ${ }^{6}$ con una matrícula mucho más acotada (aproximadamente 300 estudiantes) y una escuela con un modelo organizacional alternativo. Todas las escuelas pertenecen al sector estatal.

En relación con la selección de los docentes se consideró que estuvieran representados ambos géneros y que dictaran distintas asignaturas, y en diferentes años de la escolaridad. Las entrevistas se estructuraron a partir de la discusión de algunos tópicos comunes ${ }^{7}$ y fueron realizadas y grabadas (con consentimiento informado) a través de plataformas virtuales.

\section{Escenario de la educación secundaria en el que se produce la pandemia: permanencia y alteración de las condiciones de escolarización}

La Ley de Educación Nacional (2006), al establecer el principio de obligatoriedad de la Escuela Secundaria y promover políticas para la inclusión educativa desde la perspectiva del derecho a la educación, puso en evidencia -al tiempo que intentó modificar- la matriz selectiva del nivel. Las transformaciones curriculares, los programas ministeriales, el nuevo régimen académico han formado parte de políticas para mejorar el acceso, la permanencia y el egreso de la población estudiantil. Las condiciones de escolarización que estas políticas intentan promover entran en tensión con la estructura organizacional de la escuela secundaria y con los rasgos, aún vigentes, de su cultura selectiva.

Diversas investigaciones han dado cuenta de las dificultades que se presentan al poner en práctica políticas educativas tendientes a fortalecer la inclusión de nuevos sectores sociales en escuelas secundarias, poniendo de manifiesto tensiones entre los propósitos de las políticas y las respuestas institucionales. Coincidimos con que «el encargo social con el que estas instituciones fueron creadas no contemplaba la ética de la inclusión, sino más bien, todo lo contrario» (Furlán, 2015:13). Con el agravante de que las nuevas escuelas fueron creadas a imagen y semejanza de las existentes arrastrando más vicios que virtudes. En efecto, han ido variando las funciones de la escuela secundaria, han cambiado las poblaciones que a ella asisten, sin embargo, su expansión se dio en base al mismo modelo institucional (Acosta, 2012; Terigi, 2008). Se produce así, un desacople entre los propósitos de las políticas, los dispositivos y la cultura escolar.

La experiencia escolar se lleva a cabo bajo determinadas condiciones de escolarización gestadas en la trama entre aspectos normativos (nuevos y preexistentes que, aunque reemplazados, perviven en las prácticas), prácticas pedagógicas y la estructura organizativa que da cuenta del formato escolar (Rockwell, 1995).

Por su parte, la gramática escolar, o sea el «conjunto de estructuras, reglas y prácticas que organizan la labor cotidiana de la instrucción en las escuelas, entre las que pueden señalarse la graduación de los alumnos por edades, la división del conocimiento por materias separadas y el aula autónoma con un solo maestro» (Tyack y Cuban, 1997) producen determinadas experiencias escolares (tanto para docentes como para estudiantes) delimitando un campo de acción. Esta gramática actúa, al igual que la gramática de la lengua, de manera implícita e 
irreflexiva, estructurando nuestros modos de actuar y pensar en la escuela. Dicen Tyack y Cuban:

\begin{abstract}
Administradores, maestros y discípulos aprendieron cómo trabajar según este sistema; en realidad la gramática de la escolaridad pasó a ser simplemente la forma en que trabajan las escuelas; con el tiempo y el público, ya adoctrinado en el sistema, llegó a suponer que esa gramática encarnaba los rasgos necesarios de una «verdadera escuela». A pesar de las leyes, la costumbre institucional y las creencias culturales actuaron en conjunto para mantener en su lugar la gramática de la escolaridad. (1997: 210)
\end{abstract}

El peso estructural de la gramática explica por qué, si bien el discurso oficial (nacional y jurisdiccional) propone lineamientos de política educativa orientados al derecho a la educación y la inclusión, permanecen sedimentos de la matriz tradicional (Alterman y Coria, 2015). En el escenario actual, se han desestructurado principios básicos sobre los que se organiza la escuela y el trabajo de enseñar: separación y ordenamiento funcional del espacio, la presencialidad y la simultaneidad, el supuesto de homogeneidad y la estructuración del grupo clase, entre otros. Veremos entonces cómo se expresa el peso de lo estructural en contextos de desestructuración organizativa de la escuela.

En efecto, el ASPO ${ }^{8}$ vino a alterar los modos de organizar el espacio, el tiempo y el trabajo de docentes y estudiantes. Se han difuminado los límites entre lo público y lo privado, entre lo escolar y lo doméstico, se han alterado los modos de agrupamiento y se han diversificado las cronologías de aprendizaje y con ellas las trayectorias escolares. Sin embargo, la gramática de la escolaridad sigue teniendo fuerza configurativa en la experiencia escolar. Los cambios en las condiciones de enseñanza ponen al descubierto tensiones entre condiciones y lineamientos de política, y las decisiones para la continuidad pedagógica se configuran como parte de esta trama.

\title{
El trabajo docente ante el cambio de condiciones de escolarización. Reconfiguración de la enseñanza, saberes e intensificación
}

El trabajo docente se comprende en relación con sus condiciones y determinaciones sociales, políticas, económicas e ideológicas. Retomando las palabras de Jaume Martínez Bonafé, para entender la configuración del trabajo docente es necesario abordar el «conjunto de condiciones económicas, sociales y culturales que regulan las prácticas de enseñanza del docente e inciden en la configuración del trabajo cotidiano en la escuela» (Martinez Bonafé, 1999: 82). Asimismo, la enseñanza es una actividad laboral que, para su desarrollo, necesita de una energía vital a la que se le han incorporado conocimientos y destrezas específicas (Martínez Bonafé, 1999). Es una tarea regulada por las políticas (en la actualidad conviven algunas coyunturales orientadas a la continuidad pedagógica, a los contenidos a ser enseñados y a las formas de evaluar y calificar con otras de mediano y largo plazo como las que estructuran el puesto de trabajo docente, los modos de designación, etc.) que establecen condiciones de posibilidad. Además, la relación entre el docente y el alumno está mediada por lo institucional que interviene en la estructuración del puesto de trabajo. Trabajar en una escuela supone formar parte de una «cultura escolar» con creencias, mentalidades, prácticas y sentidos arraigados en la comunidad educativa que 
influyen en las formas específicas que adquiere el trabajo docente. Esta dimensión también asume nuevas formas y modos de hacerse presente ante la interrupción de la convivencia en las escuelas.

En el escenario actual el trabajo docente se encuentra reconfigurado en sus condiciones de existencia. En él, condiciones, saberes y cualificaciones se han visto alterados. El espacio-tiempo de realización del trabajo ha pasado del espacio institucional al ámbito privado del hogar, en donde el control administrativo sobre el trabajo ha adoptado nuevas configuraciones a ser exploradas. Estar en una escuela permitía observar la sucesión, al mismo tiempo, de diferentes hechos y situaciones en contextos interpersonales heterogéneos y cambiantes. Asimismo, el tiempo monocrónico ha sido reemplazado por una reconfiguración del mismo para atender a lo «situacional». La dimensión subjetiva del tiempo vivido en la escuela ha mutado.

Sin embargo, la irrupción de este escenario alteró las condiciones de escolarización y del trabajo docente sin que se haya modificado normativamente la organización del puesto de trabajo. Este sigue organizado a partir de la designación por horas cátedra para la enseñanza disciplinar por año escolar conforme a la organización clasificada del currículum, y bajo la concepción de trabajo individual (Terigi, 2012), poniendo límites a la toma de decisiones para la continuidad pedagógica, tanto a nivel institucional como de las tareas de enseñanza. Los directivos rápidamente replicaron esta organización en la creación de aulas virtuales considerando el docente a cargo según año y materia, utilizando principalmente Classroom, Drive y Facebook.

Un escenario de mayor flexibilización de los tiempos frente a estudiantes abrió la posibilidad de encontrar tiempos para la realización de reuniones virtuales de equipos docentes. ${ }^{9}$ Sin embargo, los saberes y las tradiciones arraigadas del trabajo individual continuaron restringiendo la construcción de consensos para proyectos colaborativos. Los intentos de trabajar de manera colaborativa han sido costosos, pues supone revertir modos organizativos y culturales del trabajo docente en escuelas secundarias. En las escuelas que contaban con la estructura de departamentos por áreas esta tarea se vio más facilitada. No obstante, en las distintas escuelas primó el trabajo según los compartimentos estancos de las asignaturas, con poca articulación de las propuestas de enseñanza y una multiplicidad y diversidad de propuestas, plataformas, metodologías y criterios utilizados por cada docente. Si bien los equipos directivos y las coordinaciones de departamentos -en los casos existentes- impulsaron algunas propuestas articuladas, la concepción de trabajo individual del puesto de trabajo y la diversidad de recursos disponibles dificultó mayores articulaciones. En las escuelas con departamentos de área consolidados, se lograron mayores avances en un trabajo colaborativo. Las desigualdades de recursos institucionales y la diversidad de culturas escolares se hacen más presentes en este contexto.

Asimismo, la imposibilidad de mantener horarios preestablecidos de clases flexibilizó e intensificó los tiempos de trabajo. Si bien, el tipo de contratación docente por horas cátedra, implicó históricamente que el docente realice un sinnúmero de tareas no reconocidas ni remuneradas (planificación, correcciones, etc.), la situación actual profundizó esta situación haciendo que todo tiempo en el hogar sea tiempo potencial de trabajo. Los tiempos se han desconfigurado. Algo tan estático y permanente como el horario escolar y la simultaneidad de la 
enseñanza, se vieron interrumpidos y las reglas del uso del tiempo pasaron a ser situacionales y diversas. La alteración del tiempo escolar, principalmente para los adolescentes, rompió con el horario escolar y con la duración de la semana. Los docentes aducen recibir mensajes en cualquier horario del día e inclusive los fines de semana, dificultando la posibilidad de desconectarse del Whatsapp.

$\mathrm{Si}$ bien no todos los docentes utilizan las mismas estrategias, algunos delimitan más los tiempos que otros. Encontramos en la mayoría de los docentes entrevistados una apertura y flexibilización de los tiempos en función de comprender la situación de los estudiantes, quiénes también vieron reconfigurados sus tiempos y espacios. No todos cuentan con un dispositivo y la conectividad necesaria en los «tiempos de trabajo». En hogares con varios hermanos que antes podían tener clases en simultáneo en distintas aulas, al compartir el mismo dispositivo la posibilidad se ve obturada. Si los padres deben cumplir con «Home office» las condiciones se tornan aún más complejas.

De este modo, el traslado de la enseñanza a los hogares subvirtió las condiciones -materiales y simbólicas- de las formas como se llevaba a cabo el trabajo de enseñar de los docentes y de aprender de los estudiantes, obligando a reinventar las prácticas, no sólo porque es necesario hacer uso de nuevos entornos y modalidades de enseñanza, sino porque fue necesario diversificar las estrategias de acuerdo con las condiciones de conectividad. De los relatos de los docentes se puede apreciar una sobrecarga de tareas, tanto por la prolongación del tiempo de dedicación individualizada a los estudiantes, como por la variedad de medios a través de los cuales cada estudiante se comunica: correo electrónico, Whatsapp, Classroom, Facebook, Instagram.

La implementación de diversas estrategias y medios de comunicación dan cuenta del intento de llevar a cabo la enseñanza con encuentros sincrónicos, pero contemplando los tiempos diversos de los estudiantes. En este sentido, se configura lo que podemos llamar una enseñanza diferida. Por su parte, los encuentros sincrónicos pueden sostenerse sólo en ocasiones, alterando el encuentro en simultáneo del grupo clase, disociándose así el momento de «transmisión»y de «recepción». Las modificaciones de la programación de la enseñanza, la diversificación de estrategias, el diseño de actividades para la modalidad virtual y el seguimiento personalizado de estudiantes al ver interrumpida la posibilidad de retroalimentación recíproca inmediata, abren para los docentes un escenario de complejidad y desafíos.

Cada docente dispone de horas de clases según la carga horaria presencial de los estudiantes en las asignaturas. Un mismo docente puede trabajar en varias escuelas atendiendo a un gran número de estudiantes a través de diversas plataformas. La ruptura de la simultaneidad genera nuevas demandas a los docentes. Les toca resolver situaciones individuales cuando antes lo hacían en el grupo clase. La economía de esfuerzos que permite el trabajo grupal y la enseñanza simultánea se ve afectada.

Los directivos desde su lugar de coordinación advierten la complejidad de la situación e intentan ofrecer orientaciones atendiendo al escenario actual. De los testimonios se aprecia que los equipos de dirección, atento a las orientaciones de la «continuidad pedagógica» ponen énfasis en la comunicación con los estudiantes y en redefinir los exigibles. El seguimiento y la atención de las trayectorias individuales de los estudiantes no queda acotada a situaciones 
particulares, sino que se vuelve la norma. Si bien es valorada por los docentes produce aún más intensificación laboral. La organización de la recepción de los trabajos (que llegan por distintos medios y formatos) y la sistematización de la información acerca de la situación de cada estudiante también implica otros tiempos, herramientas y conocimientos.

La experiencia escolar que se reconstruye de los registros muestra que el trabajo especializado acerca de la enseñanza requiere de condiciones y saberes nuevos bajo condiciones muy distintas a las de la educación presencial. En este sentido, nos interesa referir lo que Flavia Terigi (2012) denomina saber por defecto. Con una analogía con el software, la autora explica que los saberes por defecto, producidos en determinadas condiciones del sistema educativo, son los que los docentes tienen disponibles y que usarán automáticamente (reproduciendo) si no se presentan o se producen otros saberes. Como lo fuimos desarrollando anteriormente estos saberes se basan en la concepción individual del trabajo docente, en la sectorialidad del conocimiento, en la enseñanza simultánea y en la concepción monocrónica del aprendizaje. Además, y esto se vuelve central en este escenario, estos saberes se caracterizan predominantemente por la lógica de transmisión propia del mundo predigital, lo que implica que no todos los docentes contaban con los saberes necesarios para utilizar las $\mathrm{TICs}^{10}$ en la enseñanza. En el escenario actual tuvieron que aprender al mismo tiempo que producían propuestas de enseñanza en entornos virtuales. Los equipos docentes de las escuelas muestran que hay una gran disparidad de situaciones dependiendo de la edad, del manejo de la tecnología, del acercamiento previo en el contexto del plan Conectar Igualdad. ${ }^{11}$

Como da cuenta la historia de la incorporación de la tecnología a la enseñanza en el país, la misma supone un cambio cultural en etapas. La peculiaridad de la situación actual es que no hubo opción. Esta situación, además de la presión que generó, implicó una gran variedad de situaciones y también de propuestas de enseñanza para los estudiantes de acuerdo con las posibilidades de cada docente.

\section{Relaciones entre la desigualdad social y educativa: la diversificación de la experiencia escolar}

Como lo venimos señalando, la interrupción de la presencialidad en las escuelas puso en evidencia desiguales condiciones de escolarización. Los saberes acumulados y la experiencia pedagógica en el uso de la tecnología, a partir del programa Conectar Igualdad, no lograron traducirse en una institucionalización de prácticas de enseñanza con tecnología en las escuelas. En parte por la interrupción del programa que limitó dar continuidad y estabilidad a la producción de saberes pedagógicos necesarios. Aquellos docentes que pudieron asistir a capacitaciones, que lograron experimentar unidades didácticas con el uso de tecnología, hoy se encuentran en mejores situaciones para afrontar el desafío de enseñar en entornos virtuales. Esta heterogeneidad produce efectos desiguales entre escuelas y entre docentes.

Además, las condiciones de la escolarización para hacer posible la continuidad pedagógica pusieron al descubierto las desigualdades derivadas del acceso diferencial a internet, a dispositivos tecnológicos y a los usos de las tecnologías digitales (Dussel, 2004) en diálogo con las desigualdades preexistentes producto 
de la estructura social de nuestro país (Kessler, 2014). De acuerdo con la Encuesta realizada por UNICEF:

\begin{abstract}
el $18 \%$ de los y las adolescentes no cuenta con acceso a Internet en sus hogares, un porcentaje que se incrementa al $21 \%$ entre estudiantes de escuelas estatales y que afecta al 28\% de los jóvenes entre 13 y 17 en hogares destinatarios de la Asignación Universal por Hijo (AUH). Asimismo, el 37\% de los encuestados afirma que no dispone de una computadora o tableta para realizar sus tareas escolares, aumentando al $44 \%$ de los estudiantes de establecimientos estatales y al $53 \%$ de quienes perciben la AUH (UNICEF, 2020: 14)
\end{abstract}

En este mismo sentido, los resultados preliminares de la Evaluación Nacional del Proceso de Continuidad Pedagógica realizada por el Ministerio de Educación de la Nación, ${ }^{12}$ dan cuenta de limitaciones y desafíos para la continuidad pedagógica. Directores de las escuelas de todos los niveles del sistema educativo del país señalan: 1) limitaciones o falta de conectividad a Internet (88\%); 2) limitaciones en el acceso o falta de recursos electrónicos (82\%); 3) falta de experiencia en el uso de recursos electrónicos y digitales con fines pedagógicos (54\%). Alrededor de la mitad de los equipos directivos hizo referencia también a problemas asociados a la falta de conocimientos de las familias para ayudar a las y los estudiantes (48\%); a las dificultades en la organización de los horarios familiares para el estudio o la comunicación con las y los docentes (47\%); y a las dificultades socioeconómicas de las y los estudiantes (45\%). Estos ítems/ indicadores también reflejan parte de las restricciones que tienen los docentes para llevar adelante su trabajo. Asimismo, los Resultados preliminares de la Encuesta sobre Enseñanza y Aprendizaje en Tiempos de Cuarentena publicados por el Observatorio Interuniversitario de Sociedad, Tecnología y Educación (OISTE, 2020), dan cuenta de que cada quien recurrió a lo que tenía a mano para continuar el diálogo con sus estudiantes: WhatsApp, correo electrónico, YouTube, Moodle, Google Classroom, Zoom, Jitsi, Meet y más. Las plataformas y aplicaciones se convirtieron en una herramienta para subir actividades, establecer comunicaciones, dar orientaciones, organizar encuentros sincrónicos.

Estos datos en el contexto en el cual el acceso -o no- a las TICS es condición de escolarización, advierten de las formas que asumen las relaciones entre desigualdades sociales y escolares. Empieza a vislumbrarse un nuevo tipo de capital cultural necesario para desempeñarse en el nuevo escenario escolar, capital que se encuentra desigualmente distribuido (Bourdieu, 1988).

El Estado (nacional y jurisdiccional), las escuelas, los trabajadores de la educación y las familias han puesto recursos para hacer posible la continuidad pedagógica. Sin embargo, estas condiciones demostraron, tempranamente, los efectos de la desigualdad social en la desigualdad educativa. Ya existía, previo a la pandemia, una profunda desigualdad en el acceso a los recursos digitales, a dispositivos, conexión a internet, a saberes específicos para explorarlos (Magnani, 2020). Desigualdad que fue profundizada y consolidada por la desarticulación del Programa Conectar Igualdad en los últimos cuatro años.

Por otra parte, la experiencia escolar es mucho más que la transmisión de contenidos escolares. Engloba saberes, recursos, identidades, sentidos propios de las culturas escolares, localización de la escuela, su historia, etc. En sentido amplio, la irrupción de la pandemia encontró a las escuelas en una situación 
de desigualdad y heterogeneidad para abordar la virtualización de la enseñanza, aunque sólo sea para la transmisión de contenidos escolares (ya que esta modalidad dificulta la inclusión de otras dimensiones de la experiencia escolar). No todos los estudiantes y profesores contaban con los saberes para poder utilizar las TICs, ni con las mismas condiciones de conectividad y equipamiento en los hogares. En efecto, para enseñar, aprender y seguir conectados es necesario pagar internet y contar con algún dispositivo. Por tanto, las comunidades educativas reclaman el papel del Estado en garantizar la conectividad como un derecho.

De los testimonios que hemos recogido se trazan experiencias escolares diversas y desiguales. Estudiantes que nunca se han conectado, estudiantes con esporádica conexión con los docentes y estudiantes que han llevado con cierto ritmo las propuestas, dan cuenta de al menos tres escenarios y dentro de cada uno sus matices. ${ }^{13}$ Así, las experiencias escolares se diversifican, hay quienes pueden participar de las actividades virtuales y quienes tendrán que trabajar con los cuadernillos, hay quienes tendrán la posibilidad de realizar actividades sincrónicas y quienes solo podrán utilizar los dispositivos para bajar las actividades, resolverlas y luego enviarlas.

Es un escenario que visibiliza el efecto de las desigualdades sociales sobre la población estudiantil. La alteración de las condiciones para aprender en dónde la enseñanza se encuentra mediada por dispositivos, plataformas y aplicaciones ha dejado afuera a muchos estudiantes. Ya no sólo se trata de segmentación o fragmentación entre escuelas, sino que al interior de una misma escuela, las distintas posibilidades de conectividad, de espacio, de organización familiar, y de capitales culturales fue generando desiguales experiencias de escolaridad. Los directivos retoman testimonios de estudiantes que dan cuenta de la capilaridad que adopta la desigualdad.

\section{Un cierre incierto y con final abierto}

El cierre de las escuelas, el establecimiento de la continuidad pedagógica y la decisión de trasladar la enseñanza presencial a la enseñanza en los hogares (principalmente mediada por tecnologías) reconfiguró las bases del trabajo de enseñar y las condiciones para aprender de los estudiantes. Es un escenario inédito por su escala y por su impacto social, cultural y educativo. La excepcionalidad que este tiempo histórico presenta nos impulsó a conocer desde las voces de directivos y docentes las características que asume el trabajo de enseñar, las dificultades que fueron sorteando, las necesidades que se les presentaron, las demandas y los desafíos a corto y mediano plazo. Nos interesó poder apreciar el peso de la gramática de la escuela secundaria en esta nueva modalidad de enseñanza, los efectos de la modificación de las condiciones históricas de escolarización y en particular, en el trabajo docente. En este sentido, fuimos observando cómo se fueron configurando diversas y desiguales experiencias escolares debido al acceso desigual de docentes y estudiantes a las TICs, a la conectividad y a los saberes para trabajar en este nuevo escenario.

De esta manera, pudimos observar como la interrupción de la presencialidad reconfiguró el trabajo docente modificando condiciones, saberes necesarios, recursos y modos de enseñar. La experiencia escolar de docentes y estudiantes en el ámbito del hogar, visibiliza aún más las desigualdades sociales de origen. No 
obstante, la gramática de la escolaridad sigue teniendo fuerza configurativa tanto para docentes como para los estudiantes, por la persistencia de la organización del currículum, el puesto de trabajo docente y los saberes pedagógicos disponibles.

El uso de la tecnología al mismo tiempo acerca y aleja, posibilita la continuidad del vínculo y la enseñanza, pero, en la medida que está desigualmente distribuida, afianza las desigualdades configurando diversas experiencias escolares. Las nuevas condiciones para la enseñanza, orientadas por la política de continuidad pedagógica y por las decisiones de los actores institucionales más basada en sostener vínculos y en el seguimiento individualizado de les estudiantes, altera la economía de esfuerzos que supone la simultaneidad. El trabajo se intensifica porque requiere de saberes nuevos, porque el contexto del dispositivo escolar ha mutado y porque se producen nuevas demandas asociadas al trabajo individualizado con los estudiantes y colaborativo con el resto de los docentes. A su vez, los registros del modo cómo transcurre la experiencia escolar hoy permiten advertir el costo humano y emotivo de las desigualdades.

Como contracara, la visibilidad de estas desigualdades permitió que los docentes puedan cambiar algunas formas de trabajo, buscar otros recursos y, sobre todo, construir una forma personalizada de acompañar a los estudiantes.

\section{Referencias}

Alterman, N. y Coria, A. (coord.) (2015). Cuando de enseñar se trata. Estudios sobre las condiciones en que ocurre la transmisión en la escuela. Córdoba: Editorial Brujas.

Bourdieu, P. (1988). Los tres estados del capital cultural. En Sociológica, UAMAzcapotzalco, N ${ }^{\circ}$, 11-17.

Dussel, I. (2004). Desigualdades sociales y desigualdades escolares en la Argentina de hoy. Algunas reflexiones y propuestas (Documento preparado para el Ministerio de Educación de la Nación). Buenos Aires: FLACSO.

Furlán, A. (2015). Prólogo. En Alterman, N. y Coria, A. (coord.) Cuando de enseñar se trata. Estudios sobre las condiciones en que ocurre la transmisión en la escuela. 13-15. Córdoba: Editorial Brujas.

Kessler, G. (2014). Controversias sobre la desigualdad. Argentina, 2003-2013. Buenos Aires: Fondo de Cultura Económica.

Martínez Bonafé, J. (1999). Trabajar en la escuela. Buenos Aires: Miño y Dávila Editores.

Magnani, E. (2020). Educación y tecnologías. Adentro de la caja. En Dussel, I; Ferrante, P y Pulfer, D (comp.) Pensar la educación en tiempos de pandemia. Entre la emergencia, el compromiso y la espera. 85-99. Buenos Aires: UNIPE.

Rockwell, Elsie. (1995). De huellas, bardas y veredas: una historia cotidiana de la escuela en La escuela cotidiana. Ciudad de México: Fondo de Cultura Económica.

Terigi, F. (2008). Los cambios en el formato de la escuela secundaria argentina: por qué son necesarios, por qué son tan difíciles. Propuesta Educativa (No 29) - 63-71.

Terigi, F. (2012). Los saberes docentes. Formación, elaboración en la experiencia e investigación (Documento Básico para el VIII Foro Latinoamericano de Educación Saberes docentes: Qué debe saber un docente y por qué). Buenos Aires: Santillana.

Tyack, D. y Cuban, L. (1997). En busca de la utopía. Un siglo de reformas en las escuelas públicas. Ciudad de México: Fondo de Cultura Económica. 


\section{Notas}

1 La provincia de Buenos Aires se encuentra organizada en 25 regiones educativas; la Región IX está conformada por los partidos de San Miguel, José C.Paz, Malvinas Argentinas y Moreno; siendo la primera cabecera de la región. Esta región forma parte del segundo cordón del Conurbano Bonaerense

2 Sin embargo hubo escuelas que permanecieron abiertas garantizando el servicio de comedores escolares, entrega de bolsones de comida y los cuadernillos elaborados para apoyar la continuidad pedagógica

3 La continuidad pedagógica es una estrategia que ya ha sido utilizada en ocasiones como durante la Gripe A en todo el país. En la provincia de Buenos Aires, además el plan de continuidad pedagógica está plasmado en el reglamento general de escuelas (Decreto 2299/11). Sin embargo, en esta norma este plan parece estar pensado para garantizar el aprovechamiento de la jornada escolar, no para situaciones de interrupción prolongada de la escolaridad. En esta misma dirección el Régimen de la escuela secundaria de la Provincia de Buenos Aires (Res 587/11) establece el diseño de Actividades institucionales para el aprovechamiento pleno de la jornada escolar, pensadas para resolver la cuestión de las horas libres ante el ausentismo de profesores.

4 Más adelante, y ante la prolongación de la interrupción de la presencialidad se elaboraron nuevas orientaciones vinculadas al currículum y a la evaluación, la acreditación, la calificación y la promoción

5 «Como se planteó oportunamente, la prioridad ha sido el cuidado y la preservación de los vínculos de todos/as y cada uno/a de los/as bonaerenses, el cuidado y la preservación de los lazos vinculares entre los estudiantes, sus familias y todas/os las/os docentes del sistema educativo, incluyendo a todos los niveles de gestión» (Circular Técnica Conjunta $\mathrm{N}^{\circ} 1$, Dirección de Educación Secundaria, Dirección de Educación especial, Dirección de Educación Técnica)

6 En la Provincia de Buenos Aires se conoce como escuelas conformadas a aquellas escuelas secundarias que surgen de la transformación del $3^{\circ}$ ciclo del EGB en Escuela Secundaria Básica para luego dar lugar a una escuela secundaria completa, en el marco de la Ley Nacional de Educación 26.206. Suelen ser escuelas de tamaño pequeño que comparten el edificio con una escuela primaria.

7 Algunos de estos tópicos de indagación fueron: cómo se organizaron las escuelas al momento de la interrupción de la presencialidad, estrategias de sostenimiento del vínculo pedagógico y acompañamiento de trayectorias escolares, las propuestas de enseñanza y la organización de la tarea docente y el impacto de las desigualdades.

8 Mediante el Decreto $\mathrm{N}^{\circ} 297 / 20$, se dispuso el «aislamiento social, preventivo y obligatorio», durante el 20 y el 31 de marzo del 2020 para los y las habitantes del país y para las personas que se encontraran transitoriamente en él.

9 Sin olvidar que esto también suma una nueva tarea antes no prevista al trabajo docente.

10 Abreviación para las Tecnologías de la Información y las Comunicaciones. Es decir, todas esas tecnologías que nos permiten acceder, producir, guardar, presentar y transferir información. Ellas están en todos los ámbitos de nuestras vidas, en nuestra vida social, familiar y escolar.

11 El Programa Conectar Igualdad se crea bajo el decreto presidencial N459/10 que define una política de inclusión digital para la educación pública y establece la distribución de computadoras portátiles en escuelas de educación secundaria, institutos de formación docente y escuelas especiales.

12 A través de la Secretaría de Evaluación e Información Educativa y en el marco de un acuerdo del Consejo Federal de Educación (Resolución 363/20).

13 En este sentido, las últimas resoluciones del CFE y de la provincia de Buenos Aires intentan recuperar esta diversidad incorporándolas a los nuevos modos de calificación. Si bien esto podría estar vinculado con el reconocimiento de la diversidad de trayectorias para poder trabajar con ellas, también podrían construir nuevas clasificaciones que cristalicen y naturalicen estas desigualdades 


\section{Notas de autor}

Sobre la autora

Licenciada en Ciencias de la Educación (UBA) Magíster en Ciencias Sociales con Orientación en Educación (FLACSO-Argentina). Investigadora docente en el área de Educación del Instituto del Desarrollo Humano de la UNGS y docente de sociología de la Educación de la Facultad de Filosofía y Letras de la Universidad de Buenos Aires. Forma parte del del Programa de Conocimiento, Educación y Sociedad de FLACSO Argentina. Líneas de investigación son: Escuela secundaria y desigualdad; y trabajo, saberes e identidades docentes en la escuela secundaria.

Sobre la autora

Licenciada en Educación (UNGS). Docente en el área de Educación del Instituto del Desarrollo Humano de la Universidad Nacional de General Sarmiento y docente en el área de Educación de la Universidad Nacional de Hurlingham. Líneas de investigación: escuela secundaria y desigualdad.

Sobre la autora

Licenciada en Educación (UNGS), Profesora Universitaria para el Nivel Secundario y Superior en Educación (U. AUSTRAL). Docente en el área de Educación del Instituto del Desarrollo Humano en la Universidad Nacional de General Sarmiento. Líneas de investigación: escuela secundaria y desigualdad.

Sobre la autora

Licenciada en Ciencias de la Educación (UBA), Magíster en Ciencias Sociales con orientación en educación (FLACSO). Doctoranda en educación (UNCPBA). Docente e investigadora en la Universidad Nacional de General Sarmiento. Líneas de investigación: 1) escuela secundaria, políticas educativas y trabajo docente; 2) evaluación, estrategias de enseñanza y currículum. 